\section{Going with the flux}

\section{By Michael J. Haas, Senior Writer}

Researchers at Princeton University and the University of Rochester School of Medicine and Dentistry have developed an alternative approach to integrating experimental methods and computational tools to measure metabolic changes in mammalian cells. ${ }^{1}$ They have used their method to identify specific changes in cytomegalovirus- or influenza A-infected human cells, showing that fatty acid biosynthesis may play a critical role in viral infection.

Although other researchers suggested the method does not advance far beyond those used for metabolic flux profiling, Princeton is looking to protect the IP around its application in viral settings.

Metabolic flux profiling is the measurement of metabolic processes of cells. The technique is often used in chemical engineering to optimize the growth and replication of yeast or bacteria to produce desired compounds. Until recently however, in vivo metabolic profiling of disease processes was limited to analyzing biofluids obtained from animals or humans-which provides no insight into intracellular metabolism.

These profiles are "critical to understanding many indications in which flux derangement-disruptions to cellular metabolism-is implicated, such as diabetes, cancer, the Warburg effect and possibly central nervous system diseases," said Joshua Rabinowitz, professor of chemistry and integrative genomics at Princeton and leader of the research team that published the paper in Nature Biotechnology.

Recent advances have allowed the simultaneous elucidation of multiple metabolic processes within single-cell organisms growing in a minimal medium that contains only those nutrients essential to the microbes. But studying disease-related fluxes in mammalian cells has been hindered by the more complex media required to grow such cells, where the concentration of components is not always known.

Rabinowitz's team set out to overcome this challenge by combining several existing analytical techniques with a new differential equation model for data analysis. The team demonstrated proof of principle by examining the metabolic fluxes induced by CMV infection in human cells, albeit grown in a complex medium with a well-defined composition.

The group fed ${ }^{13} \mathrm{C}$-labeled nutrients-glucose and glutamine-to human lung fibroblasts infected with CMV and then monitored the kinetics of nutrient assimilation and subsequent metabolism by measuring the appearance of ${ }^{13} \mathrm{C}$-labeled metabolites with liquid chromatographytandem mass spectrometry (LC-MS/MS). Similar experiments monitored the fate of ${ }^{13} \mathrm{C}$-labeled nutrients in uninfected control cells.

Next, the team applied their differential equation model to identify which metabolic pathways were significantly altered in infected cells compared with uninfected cells.

The team found that CMV infection induced 28 distinct metabolic fluxes, including upregulation of malonyl-coenzyme A (malonylCoA), a key intermediate in fatty acid biosynthesis.

Additional in vitro experiments confirmed that fatty acid synthesis was critical to the replication of CMV. Inhibition of two enzymes involved in that synthesis-acetyl-coenzyme A carboxylase (ACC) and fatty acid synthase (FAS) - also inhibited viral replication in cells infected with influenza A virus.

This led the team to suggest in the NBT paper that inhibitors of lipid metabolism - such as those developed to treat hyperlipidemia and obesity - could have broad activity against viruses with lipid envelopes.

The group also wrote that their approach should be extendable to investigation of links between viral infection and cancer.

\section{Best flux forward}

Gregory Stephanopoulos, professor of chemical engineering at the Massachusetts Institute of Technology, said the NBT paper described "excellent experimental work and a very sound hypothesis that yielded very interesting results." But he was not certain the approach overcame any real obstacles because they used a well-defined medium.

Stephanopoulos said his group and other labs have already "shown that you can do very detailed mass balance computations and determine the metabolic fluxes in different pathways of cells in defined medium." Those computations use a technique developed by Stephanopoulos' group that is based on elementary metabolite units (EMUs). ${ }^{2}$

"In defined cell culture media, the EMU method facilitates immensely - by orders of magnitude - the measurement and mass balancing of dynamic metabolic fluxes" relative to other methods, Stephanopoulos said.

Thus, he said, unless the Rabinowitz team's method would work on mammalian cells grown in undefined medium, it offered no apparent advantages over existing methods.

Rabinowitz countered that his team's method is likely to provide substantial metabolic information even when the composition of the growth medium was not fully known. But he added: "We haven't done this, so I can't say for sure what problems would or would not arise in practice."

Technique aside, Andrew Henderson, associate professor of medicine at Boston University School of Medicine, wanted to know how metabolic changes in CMV-infected cells compared to changes in cells subjected to other stressors, such as hypoxia or treatment with a growth factor. "It wasn't clear to me if any of the observed changes in metabolism were virus specific" or more general cellular responses to stress or insult, he said.

Henderson noted that Rabinowitz's team did not perform metabolic flux analysis on influenza-infected cells to show whether influenza A virus induced the same changes as CMV.

Thus, although he agreed it is reasonable to hypothesize that blocking fatty acid synthesis inhibits replication of the two viruses, he said experiments to elucidate the inhibition mechanism were "necessary to appreciate whether this is a useful antiviral strategy." 
Henderson also pointed to existing literature-including papers cited by the NBT authors - that have already elucidated a role for phospholipids and fatty acids in viral entry and release in host cells.

John Bell, CSO of oncolytic virus company Jennerex Biotherapeutics Inc., thought the findings published in NBT might have applications related to the development of therapeutics based on oncolytic viruses because production of fatty acids is increased in tumor cells relative to normal cells.

"If we were to figure out how a particular virus induces the cell to increase conversion of glucose to fatty acids, then mutate the viral genes responsible, we would have a virus that would be able to grow in tumor cells but be growth impaired in normal cells" because of their low basal levels of fatty acid synthesis, he said.

Thus, he said, the NBT paper pointed to a difference in the interactions of viruses with normal cells versus tumor cells that "could potentially be exploited to help make selective viral therapeutics."

Bell is also professor of medicine at University of Ottawa and senior scientist at Ottawa Health Research Institute.

Indeed, Rabinowitz's team suggested that their method could be extended to other viral infections, especially viruses that have been linked to cancer. They also wrote that their method could be expanded to take into account additional factors in cellular metabolism, such as oxygen uptake and metabolism.

Laurie Tzodikov, licensing associate in the Office of Technology Licensing and Intellectual Property at Princeton, said the university had submitted patent applications for the findings reported in NBT. She declined to disclose what those patents would cover, but added that the university is pursuing out-licensing opportunities.

"We hope the integrated tools and codes will be broadly used and freely available, while the specific findings regarding viruses is a private IP matter," said Rabinowitz.

REFERENCES

1. Munger, J. et al. Nat. Biotechnol.; published online Sept. 28, 2008; doi:10.1038/nbt.1500

Contact: Joshua D. Rabinowitz, Princeton University, Princeton, N.J. e-mail: joshr@genomics.princeton.edu

2. Antoniewicz, M. et al. Metab. Eng. 9, 68-86 (2007)

COMPANIES AND INSTITUTIONS MENTIONED

Boston University School of Medicine, Boston, Mass.

Jennerex Biotherapeutics Inc., San Francisco, Calif.

Massachusetts Institute of Technology, Cambridge, Mass.

Ottawa Health Research Institute, Ottawa, Ontario, Canada

Princeton University, Princeton, N.J.

University of Ottawa, Ottawa, Ontario, Canada

University of Rochester School of Medicine and Dentistry, Rochester, N.Y. 